Planetary Decadal Survey White Paper

on

\title{
High-frequency near-surface gas measurement: an opportunity to solve puzzles in planetary atmospheric processes in Martian Methane and beyond
}

\author{
Submitting Author: \\ John E. Moores \\ York Research Chair in Space Exploration \\ jmoores@yorku.ca / +1-416-480-2735 \\ Centre for Research in Earth and Space Science \\ York University, Toronto, ON, M3J 1P3 Canada
}

Co-Authors:

Haley M. Sapers, York University, Haley.Sapers@gmail.com Dorothy Oehler, Planetary Science Institute, doehler@psi.edu

Claire Newman, Aeolis Research, claire@aeolisresearch.com

Lyle Whyte, McGill University, 1yle.whyte@mcgill.ca

Co-Signatories/Endorsers:

Sushil Atreya, University of Michigan, atreya@umich.edu

Edward Cloutis, University of Winnipeg, e.cloutis@uwinnipeg.ca

Zachary K. Garvin, Princeton University, zgarvin@princeton.edu

Raina Gough, University of Colorado, raina.gough@,colorado.edu

Hemani Kalucha, York University, hemanik@yorku.ca

Andrew C. Schuerger, University of Florida, schuerg@ufl.edu

Isaac B. Smith, York University, ibsmith@yorku.ca

Shane W. Stone, University of Arizona, stone@1pl.arizona.edu 


\section{Recent advances in understanding planetary atmospheres}

The last decade of planetary exploration has seen numerous advances in our understanding of the chemistry of atmospheres across the solar system. To give only a few examples, the MAVEN mission has revolutionized our view of upper atmospheric processes and escape mechanisms on Mars [1]. Similarly, New Horizons has discovered the importance of aerosols in controlling atmospheric temperature and escape from bodies with condensable atmospheres, allowing the retention of sufficient atmospheric gas to sustain a complex geology that goes far beyond ablative processes [2,3]. The Juno mission has revealed complexities in Jupiter's atmospheric circulation at high latitude and great depths [4]. The Trace Gas Orbiter (TGO) has allowed for unprecedented precision in measurements of individual species in the martian atmosphere [5]. The MSL mission has created the most complete record of variation in atmospheric constituents and meteorological properties near the martian surface through the REMS [6] and SAM [7] instrument suites.

However, many puzzles remain and can drive future exploration. In particular, this white paper will describe the advances that have been made in understanding a particular puzzle, methane in the martian atmosphere, as an example of what can be learned and what remains to be understood (please see section 4 for additional details on martian methane). While our understanding of methane on Mars has advanced tremendously over the past ten years, much remains to be done. And although this work includes modelling and theory, the most pressing need is for additional data in the form of high-frequency observations from the near-surface.

In this context, we define "high-frequency" as sub-diurnal and ideally comparable to the rate of measurement of meteorological parameters (temperature, humidity, wind speed and direction, e.g. [6]) that are often acquired at least hourly at surface stations on Mars. Such measurements do not need to be limited to methane. This white paper will explicitly use methane to motivate highfrequency measurement of gas concentrations in general. For instance, MSL has seen the hints of peculiar and unexpected cycles in several carbon and oxygen species including $\mathrm{O}_{2}$ and $\mathrm{CO}$ [7] that may share a related chemical solution to the methane problem. There are also comparable and tantalizing questions surrounding hydrocarbon processes on Titan [8].

Discovery often lurks in places that have yet to be explored. This is not limited to those blank spaces on the map, but also extends to blank spaces of the clock and of the calendar. (Fig. 1) A fly-by of the Earth's northern hemisphere in summer would miss the extent of the winter snow. Likewise, observing Mars only at dawn and dusk (orbital limb measurements, e.g. TGO [5]) or in the middle of the afternoon and night (sun-synchronous mapping orbits, e.g. MRO, MGS) will miss interesting diurnal effects [12]. Measurements that are only acquired every few months will miss many timescales of cycling in between. While the community currently uses modeling to fill these temporal gaps, these models can only be validated with more frequently acquired data. Surprises may await in those gaps that can address larger uncertainties in such models.

\section{It's all in the timing: what lies within the blank spaces on the clock and calendar}

Planetary atmospheres are dynamic things, changing over timescales short and long. Often it is the long-term behavior of major systems that we wish to understand. However, this long-term behavior is the sum of many short-term processes. Knowing which of these processes dominate may be difficult to tease out if our observations are not collected more frequently than the timescales over which these processes act.

To give a particularly conspicuous example: consider the total loss of water from Mars to space over geologic time. It has been very challenging to develop tight constraints on this water loss in 
numerical models that capture general parameters of the martian climate. As a result, the 2014 MAVEN mission sought to better understand the loss of water in the present era and all the processes that drive that water loss with the hope of improving those models and the estimates of past water which result. However, MAVEN discovered that the loss of water is highly dependent on several non-deterministic processes including the dust condition of the atmosphere that acts to promote or inhibit the vertical transport of water vapor up to the exobase [1,9]. As such, it is now appreciated that reconstructing the long-term background story of water loss depends at least in part on knowing the history of dust circulation on sub-annual timescales that can vary year to year.

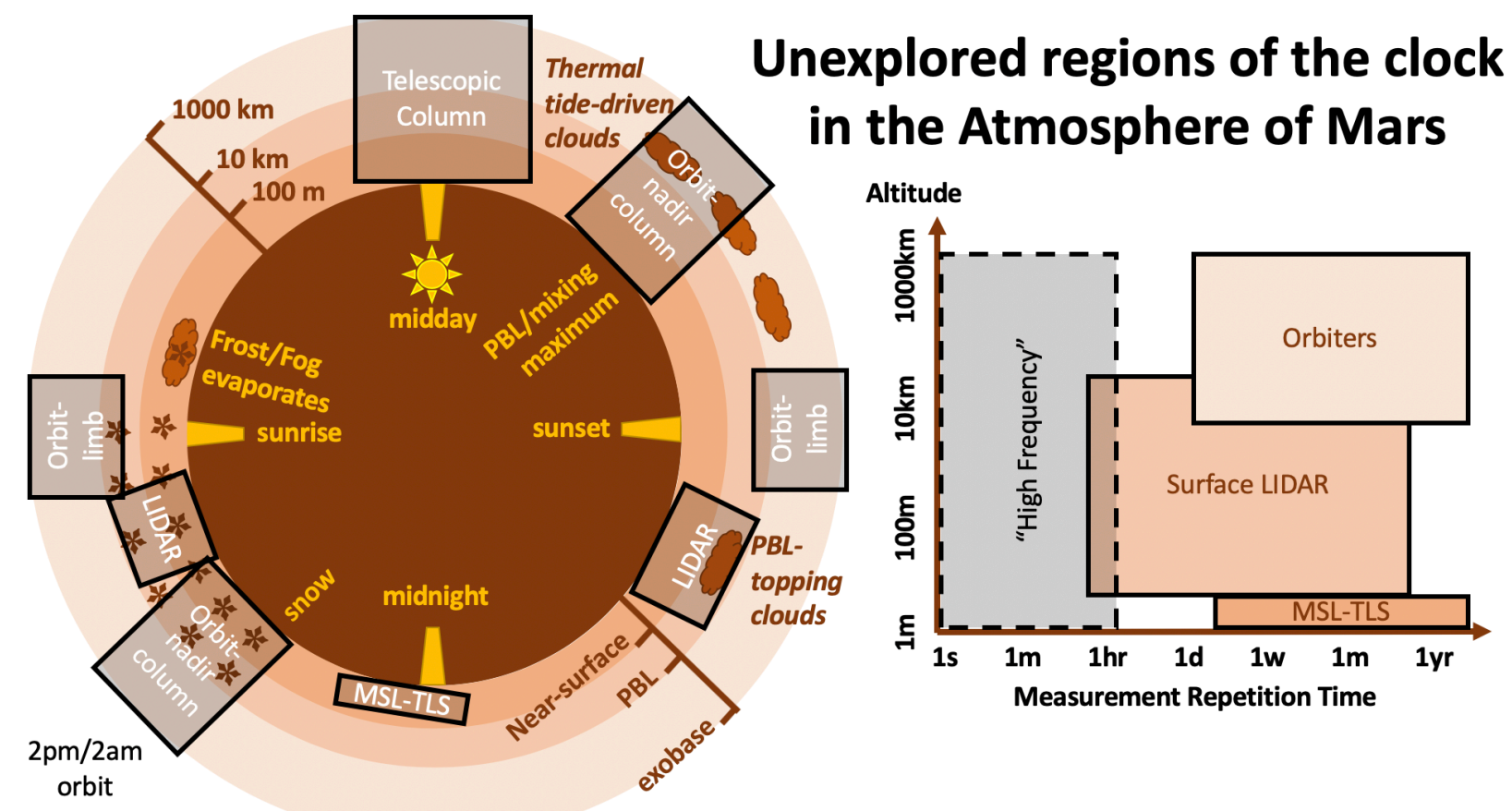

Figure 1: unexplored regions of the atmosphere and near surface in time of day (left) and in terms of repetition rate (right). Black-bordered boxes denote observations that have been made.

These include the Tunable Laser Spectrometer (TLS) of methane near midnight in the near surface; lidar observations of the PBL (all times of day, but most interesting shown [13]); orbital limb measurements near sunset and sunrise; orbital nadir-pointed column measurements in sun-

synchronous orbits; and telescopic column observations which observe the lit earth-facing hemisphere. A selection of water-condensable diurnal processes on the planet (not at all latitudes and times of year) are shown for reference. For the repetition rate at the right, SAMTLS has achieved 2 days [14], most orbiters require at least one day between limb observations of the same part of the planet at the same time and this can be longer if the geometry is not

favorable. The largely unexplored High-Frequency zone (typical of meteorological measurements) is defined as repeated observation separated by $<1$ day and preferably $<1$ hour.

The dust cycle/water cycle interaction above shows how an interannual cycle can affect a planetary system. However, by far the most common cycles in planetary systems are the result of their rotation around their axis producing a day/night (or "diurnal") cycle and their revolution around the sun, producing a seasonal cycle in insolation. So strong is this influence that most meteorological variables and chemical species concentrations in the atmospheres of planets follow repeatable diurnal and seasonal trends. These timescales therefore provide natural experiments to 
observe chemistry in the atmosphere or between the atmosphere and the surface. Only by understanding how a particular species behaves at sub-seasonal and sub-diurnal timescales can we truly understand its long-term evolution.

This effect is particularly important when considering trace and condensable species. While major atmospheric components of most planets are not easily altered by typical diurnal and seasonal processes, trace and condensable species can respond in amplified ways to changes in insolation. Consider, again, the case of water vapor on Mars. Typically, less than 10 precipitable microns (pp $\mu \mathrm{m})$ of water can be found in the atmosphere at any place or time. However, during northern summer, the exposed water ice cap begins to sublimate, pushing water concentrations above $50 \mathrm{pp} \mu \mathrm{m}$, an increase of more than a factor of five [10]. This effect can be seen beyond the concentration of water. A concurrent decrease is observed in the $\mathrm{D} / \mathrm{H}$ (deuterium to hydrogen) ratio of atmospheric water as the relatively highly fractionated atmospheric reservoir is replenished with the less heavily fractionated polar source [11] resulting in a variation of an order of magnitude in this ratio.

Seasonal effects are easier to observe than are diurnal changes. For instance, many orbiters keep sun-synchronous orbits that have advantages for spacecraft operations while prioritizing surface mapping. Such an orbit implies that the spacecraft passes over every part of the surface at only two local times of day, for instance a $2 \mathrm{PM} / 2 \mathrm{AM}$ orbit. This has advantages in making phase angles and shadows consistent to bring out details in the surface and in maximizing thermal inertia measurements for grain size determination. However, these sorts of orbits hide diurnal processes. For instance, some of the most interesting atmospheric effects including frosts and fogs are seen around the time of sunrise and sunset (figure 1). Such observations can tell us where near-surface water is relatively abundant, such as in most low-lying equatorial areas [12] and where it is absent, such as Gale Crater [12] which later turned out to be particularly arid [6].

As such, relatively spatially and temporally restricted fluctuation mechanisms can drive observable variations at planetary scales. The corollary, that observable variations at planetary scales can be explained by and are products of, as of yet, unexplored local phenomena, is the driving rational to propose high frequency atmospheric measurements

\section{Why being near the surface matters}

Note that for the martian water processes discussed above, like many other interesting chemical puzzles, it is the interaction between the surface and the atmosphere that has the greatest impact, and it is at the surface where the greatest effect would be observed. For instance, the Phoenix lander observed a diurnal cycle of water vapor within the Planetary Boundary Layer (the part of the atmosphere in direct contact with the surface that is homogenized daily by convective mixing). with water sublimating during the morning, being mixed up to altitude during the afternoon and forming clouds overnight which, in turn, snowed out [13] depositing water ice on the surface [15]. Yet, despite this visible cycling process, much is unknown about water exchange between non-ice regolith and the atmosphere and many numerical models therefore entirely neglect this source.

In planetary exploration, the atmosphere is the easiest part of a planet to examine. From even an exoplanetary distance, we can observe bulk atmospheric constituents of some planets through the light absorbed during transits. From orbit, very precise measurements of even trace gases can be obtained that are critical to understanding the global picture [5]. These observations can yield column abundances collected over footprints as small as tens of $\mathrm{km}$. They can also provide vertical profiles of the atmosphere by observing the planet's limb. But these observations are limited by topography and total absorption (opacity) and therefore can come no closer to the surface than 3- 
$5 \mathrm{~km}$ [5]. This is unfortunate since many processes may be driven by interactions with the surface, such as condensation, absorption and gas-solid chemistry, or re-supply from subsurface reservoirs of atmospheric species.

Meanwhile, sensitive measurements acquired on or near the surface (within the PBL where mixing is important) can provide key data about local processes that are too restricted to be visible from orbit. Moreover, they can observe this part of the atmosphere at any time of the day or night, which is challenging for orbital spacecraft that are limited to overflights. Indeed, such overflights are often scheduled for the same time of the day, day after day, due to the appeal of sunsynchronous orbits for mapping and spacecraft operations. This limits the ability of satellites to examine the role played by the diurnal cycle in atmospheric processes.

Though a network of surface measurements is desirable, it is also true that the effects of global processes are visible even to a single local station. On Earth, the Keeling dataset of carbon dioxide measurements in Hawai'i [16] is able to observe not only the human production of greenhouse gasses, but also the seasonal uptake and release of that carbon dioxide by the boreal forest. On Mars, the effect of differential condensation on the polar caps can be seen clearly in the volume mixing ratios of nitrogen and argon that are preferentially retained in the atmosphere and therefore concentrated as carbon dioxide is condensed at the winter pole. A close examination of the timing of this process reveals important information about atmospheric transport processes [7] that can be used to validate numerical models and improve their predictive power. In this way, a local near-surface observation provides more than just ground truth: these measurements can enhance and give context to orbital datasets.

\section{Methane as an example of where high-frequency surface measurements are key}

Methane on Mars is particularly interesting because, at least on the Earth, the vast majority of methane is produced and consumed by biological processes. On Mars, the absolute concentration of methane on Mars quite small and at no place and time greater than $\sim 45$ parts per billion by volume (ppbv) or so [17], as compared to the 2000 parts per million (ppm) concentration in our own atmosphere. However, this small amount is significant because our current understanding of the physics and chemistry of the atmosphere of Mars indicates that methane should have a lifetime of only $\sim 300$ years [18] before it is oxidized or destroyed by photolysis. This requires that the gas be constantly resupplied by some active process in the modern era for it to be visible to terrestrial telescopes and spacecraft instruments. Biology is one of those candidate processes, along with water-rock reactions [19, 20], release of ancient methane (from trapped accumulations; clathrates or degrading organic materials) and exogenic processes which could replenish a feed-stock of organic carbon through comets [21], and UV degradation of carbonaceous chondrites [22] or interplanetary dust particles [23].

As a result, many groups have attempted to measure methane on Mars either telescopically from the Earth [25, 17], from Mars orbit [26, 27, 24, 5] and from the surface [28, 29, 30]. Given the low concentration in the atmosphere, methane concentration is a challenging measurement to make that can require substantial data reduction steps. Moreover, the observations often require significant resources that results in relatively infrequent measurements, typically months apart for the same geographic location (e.g. [17, 30]). As such, only two measurements made by different spacecraft have ever sampled the same airmass at the same time [24, 29] and none have observed the evolution of a positive result over time - all detections stand as single measurements.

From these myriad observations there are two types of behavior into which the positive detections can be sorted. First, there are periodic (and typically isolated) high values, termed 
"plumes" or "spikes" by the community in which methane is detected at levels above 2 ppbv for a single measurement and may be as high as $40 \mathrm{ppbv}$ (typically disappearing by the next measurement). These have been observed by all groups except for TGO [5]. For the TLS-observed plumes, which sample a small volume of atmosphere, this could indicate an airmass with a relatively large methane concentration that briefly mixed with air near the rover before dissipating or advecting onwards with the wind. For instance, the $19.5 \mathrm{ppbv}$ "New York Times" plume was not observed by the next observation 2 days later [14].

This behavior has also been seen telescopically, however, the geographic size of these plumes [17] is more challenging to explain. Even if they disperse throughout the whole of the martian atmosphere, the amount of mass involved means that their subsequent non-detection, in the case of the Mumma plumes over 4 months, challenges our understanding of the chemistry of Mars [31]. The plumes may contain a great deal of material, perhaps as much as 19,000 tonnes in a single event [17] and are therefore of significant astrobiological interest. Yet, if the atmospheric lifetime of such an event is 300 years, then the methane released by the Mumma plume should still be visible today in measurements as a well-mixed $\sim 10 \mathrm{ppbv}$ signal throughout the whole of the martian atmosphere.

The second type of behavior is a "background" level of methane that varies with the seasons and is typically present at levels of $<1$ ppbv. Primarily described by [30], this background represents a fairly small amount of methane. Again, using a 300-year lifetime and assuming (in a maximal limiting case) that the signal is global, suggests that this background concentration is maintainable with a yearly resupply of no more than $\sim 30$ tonnes [32]. Yet the amplitude of the seasonal cycle, a factor of 3 from the lowest to the highest concentration, stands in opposition to numerical models with 300-year lifetimes [33] which predict that the gas should vary by only $20 \%$ at MSL's Gale Crater landing site. Heterogeneous release from some locations but not others [14] can allow for more variation, but unless Gale is very special, there is a missing piece to the methane puzzle on Mars.

Though some question the present measurements that claim positive detections of methane (notably, [34]), the absence of methane to the detection limits of TGO is perhaps even more puzzling. Hundreds of tons of organic carbon accrete to the surface of Mars each year as interplanetary dust particles (IDPs) [35] and this material is known to generate considerable methane in terrestrial labs when exposed to UV irradiation and atmospheric conditions similar to the martian surface $[22,36]$. This is sufficient organic carbon to generate more than two orders of magnitude more methane than the TGO detection limit. Thus, even if conversion rates are low, or if the IDP flux is overestimated, a detectable background of methane should still be present.

Taken together, all of these lines of evidence suggest that there is a missing piece to the methane cycle on Mars that strongly indicates there is new chemistry to be discovered.

\section{What could we learn from solving the methane puzzle?}

These questions will not be easily answered by more modeling. Instead, what is missing is more data that can be used to originate and test theories and validate those models. Without knowing how long the plumes last and how they rise and decay (since all are single high measurements), it is difficult to understand what processes bring them about and what processes cause them to disappear. Similarly, the only current framework that can simultaneously explain both the TGO non-detection and the TLS background [14] is able to reconcile these measurements only if methane concentrations can vary on timescales of hours. As such, high-frequency 
measurements, building upon the results of the last decade, would allow us to better understand how this gas varies on short timescales and why (e.g. Fig. 2).

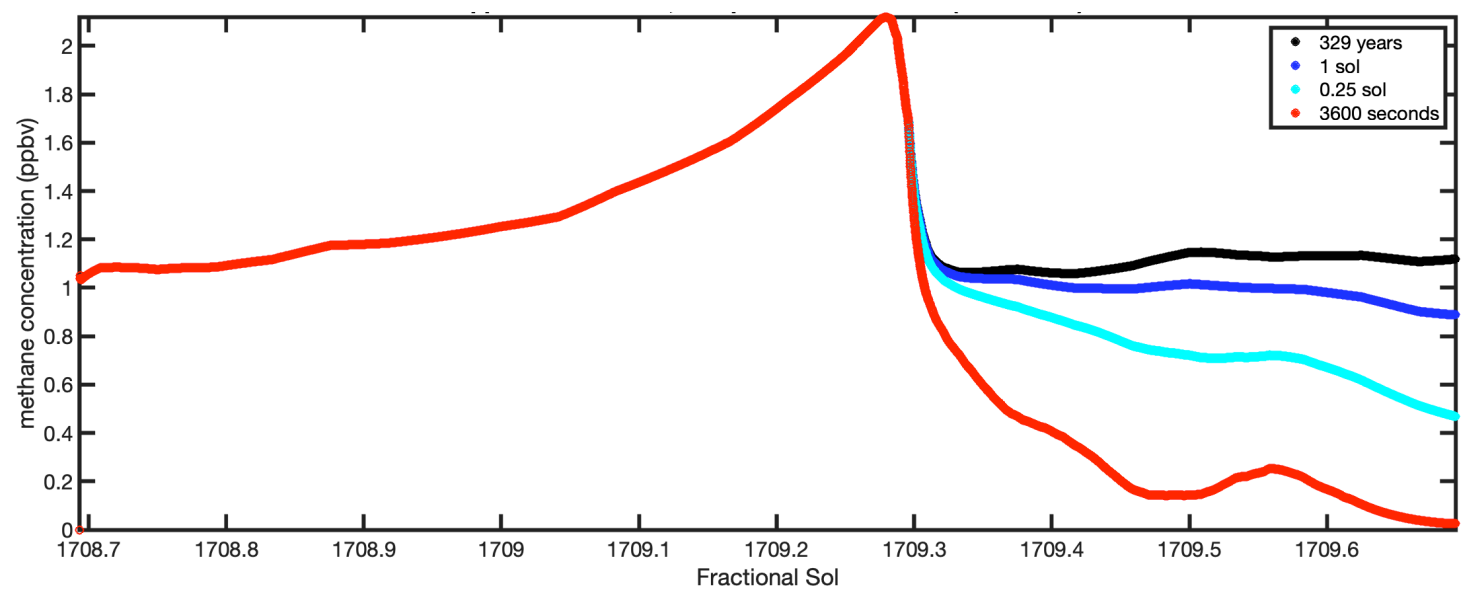

Figure 2: overnight run using the model of [32] to show how methane concentrations near the surface behave in the presence of a background 1 ppbv plume. Different lifetimes for methane are shown as different coloured traces. The way in which the methane concentration behaves in the hours following sunrise reveal the lifetime of the gas.

Additionally, the concentration of methane seen at the surface, while low, is not so tiny that it is inaccessible to modern optical spectroscopic techniques such as Integrated Cavity Optical Spectroscopy (ICOS) and the related technique Cavity Ring-Down Spectroscopy (CRDS). These new techniques have much longer effective pathlengths (up to tens of $\mathrm{km}$, compared to $16.8 \mathrm{~m}$ for the TLS) that make enrichment unnecessary at the 100s of pptv (parts per trillion by volume) level. This means that observations with these techniques are much less resource intensive and can therefore be completed more frequently.

As well, these instruments could also track other species which vary in unusual ways and whose behavior may be related to the same surface chemistry affecting methane. Notably, free oxygen $\left(\mathrm{O}_{2}\right)$ and carbon monoxide $(\mathrm{CO})$ could provide information on the action of strong oxidizing agents and surface chemistry. When plumes are present, these techniques could also be applied to any accompanying gasses (e.g. ethane) and to the different isotopologues of methane to provide additional insight into biotic vs abiotic origin. Finally, a high-frequency approach has the potential to identify locations of significant methane accumulation or production in the subsurface. These could be sites for future, detailed in-ground collection and assessment of that methane's origin and significance.

\section{Conclusions}

Methane: The measurements of the past 10 years have revealed an enticing puzzle in methane on Mars. We have some measurements, but do not know the source of this gas, nor why the concentration sometimes increases quickly (plumes) and then disappears equally quickly in a way that seems to defy our current understanding of the near surface chemistry of the martian atmosphere. In-situ high-frequency (sub-diurnal) gas concentration measurement near the surface has the potential to address this problem by detecting the onset and decay phases of the plumes that have never been observed. This observation goal would also help to provide insight into whether the methane so-generated is biotic or abiotic in origin or was created recently or in the 
distant past. Even in the absence of plumes, the rapid measurement of the evolving concentration of methane could give insight into the quantity of exhalation from the subsurface and other aspects of near-surface chemistry.

Trace gasses in general: The application of this technique to other trace gasses and processes on Mars and beyond could help to resolve other outstanding questions. Ultimately, measuring concentrations of trace species with the same frequency as we make meteorological measurements will yield rich datasets that will allow theories to be developed and tested and models to be validated that will ultimately expand our understanding of the dynamic atmospheric and geologic processes active on Mars today with implications that will constrain modern habitability.

\section{References}

[1] Jakosky, B.M. et al. (2018) Icarus 315 p 146-157 doi: 10.1016/j.icarus.2018.05.030 [2] Zhang, X. et al (2017) Nature 551 352-355. [3] Olkin, C. B. et al. (2017) Nature Astronomy (1) p 663-670. doi: 10.1038/s41550-017-0257-3 [4] Bolton, S. et al. (2017) Geophys. Res. Lett v44 (15) pp. 7663-7667 doi: 10.1002/2017GL074118 [5] Korablev, O. et al. (2019) Nature 568, 517-520 [6] Martinez, G.M. et al., (2017) Space Sci. Rev. 212.1-2: 295-338 doi: 10.1007/s11214017-0360-x [7] Trainer, M.G. (2019) JGR: Planets v124 (11) pp. 3000-3024 doi:

10.1029/2016JE006175 [8] Hörst, S.M. (2017) JGR: Planets v122 (3) p. 432-482 doi: 10.1002/2016JE005240 [9] Jakosky, B.M. et al (2019) AGU Fall Meeting abstract \#P52C-06 [10] Fedorova, A. et al. (2006) JGR: Planets v111 (E9) CiteID E09S08 [11] Villanueva, G.L. et al. (2015) Science 348 (6231) pp. 218-221 doi: 10.1126/science.aaa3630 [12] Mohlmann, D.T.F. et al. (2009) P\&SS 57 (14-15) pp.1987-1992 doi: 10.1016/j.pss2009.08.003 [13] Whiteway, J.A. et al. (2009) Science v325 (5936) pp 68- doi: 10.1126/science.1172344 [14] Moores, J.E. et al. (2019) Geophys. Res. Lett. 46 (16) pp. 9430-9438. Doi: 10.1029/2019GL083800

[15] Moores, J.E. et al. (2011) Geophys. Res. Lett. 38 (4) CiteID L04203 doi: 10.1029/2010GL046315 [16] IPCC Climate Change 2014: Synthesis Report (eds Core Writing Team, Pachauri, R. K. \& Meyer, L. A.) (IPCC, 2014) [17] Mumma, M.J. et al. (2009) Science 3235917 pp 1041-1045 doi: 10.1126/science.1165243. [18] Atreya, S.K., et al. (2007) Planetary Space Sci. 55, 358-369. [19] Oehler, D.Z. and G. Etiope (2017) Astrobiology. V17. DOI:

10.1089/ast.2017.1657 [20] Etiope, G. and Oehler, D.Z. (2019) Planetary and Space Science 168 52-61 doi: 10.1016/j.pss.2019.02.001 [21] Fries, M. et al. (2015) Geochem. Perspect. Lett., 2, 10-23. [22] Schuerger et al., (2012) JGR: Planets v117 E8 E08007 doi: 10.1029/2011JE004023 [23] Moores, J.E. et al. (2012) JGR: Planets v117 E8 E08008 doi: 10.1029/2011JE004060 [24] Giuranna, M. et al. (2019) Nature Geoscience 12 326-332 doi: 10.1038/s41561-019-0331-9 [25] Krasnopolsky, V.A. et al. (2004) Icarus 172 (2) p. 537-547 doi: 10.1016/j.icarus.2004.07.004 [26] Formisano, V. et al. (2004) Science 306 (5702) pp. 1758-1761 doi:

10.1126/science.1101732 [27] Geminale, A. et al. (2011) Planetary Space Sci. 59 (2-3) p.137148 doi: 10.1016/j.pss.2010.07.011 [28] Webster, C.R. et al (2013) Science 342 (6156) pp. 355357 doi: 10.1126/science.1242902 [29] Webster, C.R. et al (2015) Science. 347 (6220) pp. 415417 doi: 10.1126/science.1261713 [30] Webster, C.R. et al (2018) Science. 360 (6393) pp. 10931096 doi: 10.1126/science.aaq0131. [31] Lefèvre, F., Forget, F., (2009) Nature 460, 720-723. [32] Moores, J.E. et al (2019) Nature Geoscience. 12 321-325 doi: 10.1038/s41561-019-0313-y [33] Meslin, P.-Y. et al. (2011) Planet. Space Sci. 59, 247-258 [34] Zahnle, K. et al., (2011) Icarus 212, 493-503. https://doi.org/10.1016/j.icarus.2010.11.027. [35] Flynn, G.J. (1996) Earth Moon Planets 72, 469-474. [36] Keppler, F. et al. (2012) Nature 486, 93-96. https://doi.org/10.1038/nature11203. 